\title{
Recurrent venous thromboembolism- its causes, prevention and management
}

\begin{abstract}
Recurrent Venous Thromboembolism (VTE) is regarded a chronic disease, as it often recurs in spite of effective management with anticoagulant therapy including low molecular weight heparin, fondaparinux, warfarin and newer oral anticoagulant therapy. Patients with chronic recurrent VTE, develop other complications including post-thrombophlebitic syndrome and chronic stasis venous ulcers. The scientific data behind chronic VTE is sparse, and there are no standard guidelines for managing these patients. The financial and social burden of chronic VTE is enormous, with patients suffering with chronic pain, swelling and cramps leading to impaired activities of daily living and poor quality of life. Investigations to identify the underlying cause of chronic VTE, including inherited thrombophilia workup adds to the financial burden and does not help in the management of recurrent DVT. We will review the underlying causes of recurrent VTE its prevention and management.
\end{abstract}

Keywords: recurrent DVT, inherited thrombophilia, venous ultrasound, doppler studies, heparin, fondaparinux
Volume 5 Issue I - 2017

\author{
Nathan Visweshwar,' Michael Jaglal, ${ }^{2}$ Lubomir \\ Sokol ${ }^{2}$ \\ 'Department of Hematology, University of South Florida, USA \\ ${ }^{2}$ Department of Medical Oncology, Moffitt Cancer Center, USA
}

Correspondence: Nathan Visweshwar MD.,FRCPC,

Department of Hematology, University of South Florida, I3330, USF Laurel Dr Tampa, FL 336I2, USA, Tel 8I39743725,

Email nviswesh@health.usf.edu

Received: June 18, 2017| Published: July 20, 2017
Abbreviations: VTE, venous thromboembolism; DVT, deep vein thrombosis; PE, pulmonary embolism; FVL, factor V leiden; DUS, doppler sonography

\section{Introduction}

Venous thromboembolism (VTE) occurs in approximately 900,000 patients per year in the USA, resulting in 300,000 deaths from pulmonary embolism with the incidence remaining similar in the last 25 years. ${ }^{1}$ Heparin has been the treatment of choice for VTE for more than four decades. Mortality of untreated DVT is high and maximum deaths occur before or within one month from diagnosis of VTE. ${ }^{1}$ Approximately one third of patients with symptomatic VTE manifest with pulmonary embolism (PE) and two thirds manifest deep vein thrombosis (DVT) alone. Chronic thrombosis occurs in about $30 \%$ of patients and is more prevalent in the proximal veins. ${ }^{2}$ Patients with chronic changes in the deep venous system require different treatment strategy. Some of these patients may need local measures for recurrent thrombosis which may include preventive measures with initial thrombolysis, correction of the anatomical defect with intraluminal stent or other surgical interventions to prevent recurrent DVT and evaluation for systemic hypercoagulable state. Studies on deep vein thrombosis have been focused on inherited hypercoagulable state which includes a panel of tests with minimal emphasis on local factors such as the distribution, extent of thrombosis and their association with acquired systemic risk factors. ACCP Guidelines 2016 recommend long term anticoagulant therapy, irrespective of underlying inherited hypercoagulable state for patients with recurrent VTE. It is most important to exclude local cause of VTE, as most of these patients with recurrent may need local measures apart from systemic anticoagulant therapy. In this review we aim to identify pathogenesis of recurrent VTE, identify both systemic and local causes and treatment strategies to prevent and treat recurrent VTE (Figure 1).

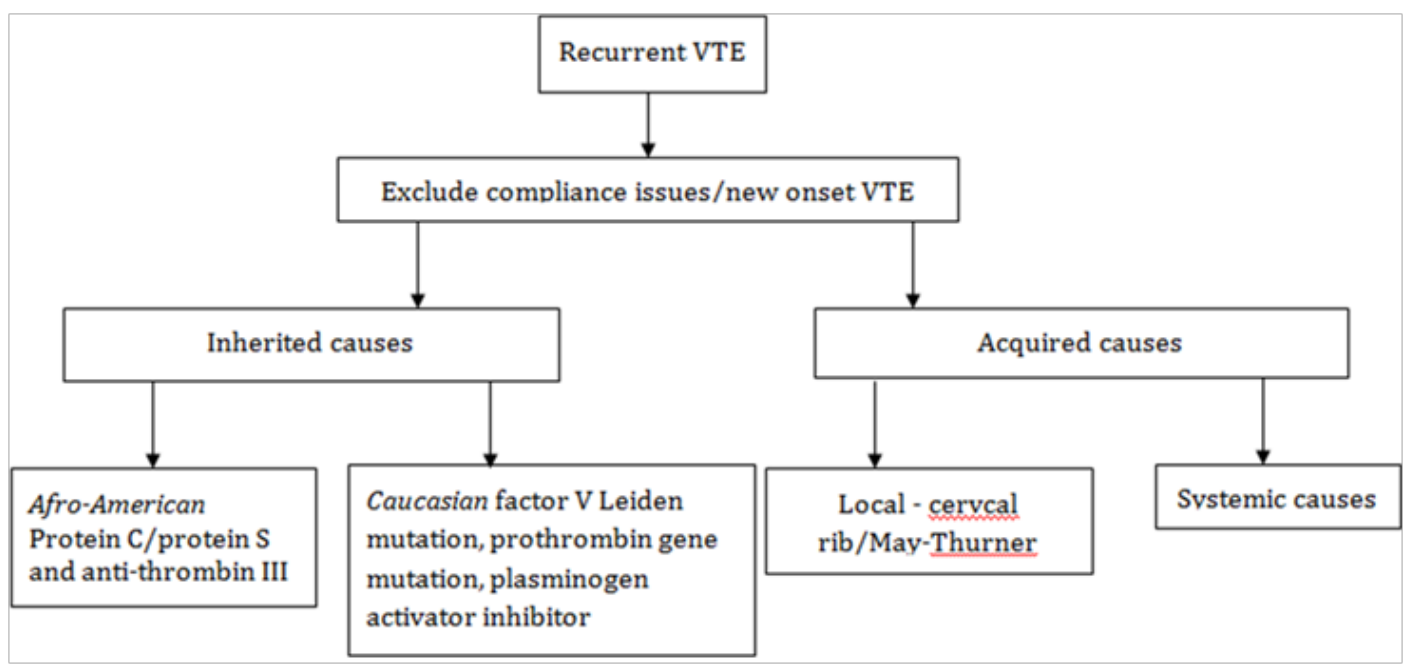

Figure I Flow chart summarizing the workup for recurrent VTE. 


\section{Recurrent VTE- causes}

Local: Paget-Schroetter syndrome is effort thrombosis of subclavian vein associated with strenuous activity of upper extremities. The proposed mechanisms include: hypertrophy of the pectoral muscles causing extrinsic compression of the vein and repetitive trauma damaging the venous wall leading to intimal damage. ${ }^{3}$ May-Thurner syndrome is the pathologic compression of the left common iliac vein by the right common iliac artery, resulting in left lower extremity pain, swelling, and deep venous thrombosis. In upper limb deep vein thrombosis $(80 \%$ of the total) was due to central venous catheters and malignancy. ${ }^{4}$ Congenital IVC anomalies including inferior vena cava atresia must be considered in young patients presenting with idiopathic DVT. ${ }^{5}$ In about $30-40 \%$ residual scar tissue is the cause of recurrent DVT.

Systemic (acquired): Predictors of recurrent DVT were reported by Heit et al. ${ }^{6}$ These characteristics included the following: type of event (PE, DVT, or both); age at incident event; sex; patient location at onset (community, community but hospitalized within the previous 90 days, hospital, or nursing home); body mass index, chronic heart disease; active malignant neoplasm with or without chemotherapy; serious neurologic disease; surgery requiring anesthesia; anesthesia (general, epidural/spinal, other); trauma requiring hospital admission; chronic lung disease; chronic liver disease; chronic renal disease and inflammatory bowel disease. The characteristics related to active malignant neoplasm, chemotherapy, surgery, anesthesia, trauma, hormone therapy, oral contraceptive use, and tamoxifen therapy were recorded as present only if documented within the 3 months prior to the VTE event. 6

Systemic (Inherited): More than $10 \%$ of the general population is affected by one or more identifiable inherited thrombophilia. ${ }^{7}$ About $90 \%$ of patients with inherited thrombophilia develop recurrent DVT. ${ }^{8}$ Estimates of the 5-year cumulative incidence of recurrent thrombotic events are around 25\%. ${ }^{9}$ Critical review of four studies highlighted hazard ratios ranging from 1.1 to 4.1 in carriers of factor V Leiden (FVL) compared with non-carriers and for carriers of prothrombin G20210A with non-carriers, hazard ratios of a recurrent thrombotic event varied from 0.9 to $4.9 .^{10,11}$ Among patients with plasma factor VIII levels above the 90th percentile, as compared with those with lower levels, the overall relative risk of recurrence was 6.7 fold. ${ }^{12}$ A meta-analysis reported the prevalence of Factor V Leiden to be significantly higher in patients with isolated DVT than in patients with pulmonary embolism (with or without DVT). ${ }^{13}$ Patients with hyperhomocysteinemia are at high risk of recurrent venous thromboembolism. ${ }^{14}$ But most studies showed little effect of carrier state of these mutations on the risk of recurrence, ${ }^{15-17}$ and Clinical factors are probably more important than laboratory abnormalities of inherited thrombophilia in determining the duration of anticoagulation therapy. ${ }^{18}$

\section{Diagnosis}

Duplex and color doppler sonography in diagnosis of recurrent DVT: Imaging techniques including compression ultrasonography, computed tomography venography and magnetic resonance imaging have been used for suspected recurrent DVT but has never been validated for this. ${ }^{19}$ Duplex and color Doppler sonography (DUS) is currently the technique of choice for the diagnosis of deep venous thrombosis (DVT) in symptomatic patients, because it is safe and cost-effective, with a very high sensitivity and specificity (96\% and $98 \%$, respectively) for the diagnosis of proximal DVT. ${ }^{20}$
Several issues regarding its method and clinical indications remain controversial, however. Isolated calf vein thrombosis does not seem to have a significant adverse outcome in the short term and scanning the calf only, in patients with localized symptoms or signs is costeffective. ${ }^{21}$ Bilateral examination is indicated in high-risk patients or when screening asymptomatic patients. ${ }^{22,23}$ Some populations of asymptomatic patients at high risk of DVT may benefit from duplex ultrasound screening. ${ }^{24}$ Recurrent thrombosis is a challenging diagnosis for all imaging modalities. Ultrasound imaging is a useful method to study changes in the venous system because it can locate a thrombus, assess the changes which occur over time and monitor the development of recanalization and reflux of blood flow. Follow up ultrasound after an year revealed changes noted in $34 \%$ with early post-phlebitic symptoms. ${ }^{25}$ The pitfalls and limitations of venous duplex ultrasound are related to vein anatomy, flow changes, technical issues, and operator expertise..$^{20,26}$

Role of ultrasonography/doppler in follow up of DVT: Currently available evidence provides no basis for using ultrasonography to guide the duration of anticoagulation, and therapy is not based on guidelines. There are few indications for the noninvasive followup of DVT during anticoagulant treatment in the absence of new symptoms. ${ }^{27}$ The error associated with ultrasound measurements of residual vein diameter, thrombus echogenicity and flow appears to be considerable. ${ }^{28}$ Based on ultrasonography patients with multiple sites of thrombosis than in those with thrombosis in a single vein segment are prone to develop recurrent thrombosis and skin damage. ${ }^{29}$ But when consecutive patients with a first episode of proximal DVT documented by Doppler ultrasound imaging were included prospectively 1 year after the diagnosis, recurrent DVT at 1year occurred in $21.9 \%$, including ipsilateral in $15.6 \%$ and contralateral in $6.3 \%{ }^{30}$

\section{Management of recurrent VTE}

Prevention: At long-term follow-up, 25 percent of patients who received recombinant tissue plasminogen activator plus heparin had symptoms of the post-phlebitic syndrome, compared with 56 percent of patients who received heparin alone. ${ }^{31}$ Rapid thrombolysis and preservation of valvular venous function in high deep vein thrombosis may prevent post-thrombotic sequelae..$^{32}$ The occurrence of severe post thrombotic syndrome after primary treatment with isolatedpharmaco-mechanical thrombolysis device is low. ${ }^{33}$ But others have not confirmed these findings. ${ }^{34}$ In a Cochrane review reduced incidence of post-thrombotic syndrome (by $1 / 3^{\text {rd }}$ ), similar effectiveness was seen with systemic thrombolytic therapy and catheter directed thrombolysis. ${ }^{35}$ The post-thrombotic syndrome occurs in almost one third of these patients and is strongly related to ipsilateral recurrent deep venous thrombosis. These findings challenge the widely adopted use of short-course anticoagulation therapy in patients with symptomatic deep venous thrombosis. ${ }^{36}$ Vitamin k antagonists, Direct oral anticoagulants and Aspirin all reduce the risk of recurrent DVT, with Vitamin k antagonists and direct oral anticoagulants being more effective than Aspirin. ${ }^{37}$

Treatment: There are no evidence based guidelines, presently available for management of chronic DVT. The following is the suggested treatment algorithm for recurrent DVT developed during anticoagulation therapy.

i. Confirm chronic DVT by compression ultrasonography

ii. Exclude non-adherence to the existing anticoagulant regimen 
iii. Commitment to long-term anticoagulation therapy

iv. The newer oral anticoagulants may be used, if there are no contraindications including renal failure

v. Enoxaparin is the drug of choice in recurrent DVT

vi. If patients develop recurrent DVT on Enoxaparin, increase of the dose by $25 \%$ he suggested

vii. If patients develop recurrent DVT on therapeutic dose of warfarin addition of aspirin or initiation of fondaparinux may be other alternatives

viii. In patients with cancer the present drug of choice is enoxaparin

ix. Appropriate management of post-phlebitic syndrome (in about $30 \%$ of patients) with graded compression stocking/elevation of the leg along with continuation of anticoagulant therapy suggested

x. IVC filters have no major role in management of chronic DVT as there is no benefit in overall survival.

Local measures: Management of May-Thurner syndrome may need interventional radiology, vascular surgery, cardiology, and vascular medicine and it is imperative to confirm the diagnosis to reduce misdiagnosis and inappropriate treatment. ${ }^{38}$ Patients with thoracic outlet syndrome including Paget-Schroetter syndrome need surgical decompression of the vascular bundle at the thoracic outlet, after initial thrombolytic therapy. ${ }^{39}$ Stenting for chronic post-thrombotic vena cava and iliofemoral venous occlusions is beneficial. Stenting to treat venous claudication, edema and recurrent venous ulcer caused by post-thrombotic chronic venous occlusions has positive clinical outcome. ${ }^{40}$ Involvement of major veins including common iliac vein lesion invariably needs to undergo stent placement. ${ }^{38}$ Surgical thrombectomy may be an effective method for treating severe acute DVT in lower extremity to prevent recurrent DVT but may lead to chronic complications including post-phlebitic syndrome. ${ }^{41}$ Thrombolysis was successful in $85 \%$ with complete clot lysis and in $>90 \%$ restored patency. No pulmonary embolism and rare bleeding complication at the catheter-insertion site were observed. ${ }^{42}$ Surgery for the deep venous must be reserved for patients who suffer from venous insufficiency with serious venous reflux and ambulatory venous hypertension. ${ }^{43}$ The prompt administration of adequate compression elastic stockings in patients with symptomatic DVT has the potential to reduce the frequency of late post-thrombotic syndrome development by half. ${ }^{44}$

\section{Discussion}

Recurrent deep vein thrombosis often occurs from noncompliance of the treatment regimen. Hence, the need to maintain the dose and duration of treatment should be stressed to prevent recurrence of DVT. Preventive measures including thrombolytic therapy of the proximal lower limb DVT is of unproven benefit. Specific therapy for local causes including stenting for May Thurner syndrome or specific therapy for cervical rib or fibrous strand may have a beneficial role. Objective testing is mandatory to confirm or refute the presence of recurrent DVT, before commitment to long-term anticoagulant therapy, as only $30 \%$ of patients with suspected recurrence have a new documented thrombotic episode. Lupus anticoagulant syndrome, underlying cancer, paroxysmal nocturnal hemoglobinuria and heparininduced thrombocytopenia may have to be excluded for appropriate management of a patient presenting with recurrent DVT, as these are definite contraindications for the newer oral anticoagulant therapy.
Endovascular surgery is of no proven benefit in patients with recurrent DVT. The role of permanent vena cava filter is debatable, as there is no proven survival benefit.

Despite considerable progress in the diagnosis and treatment of deep vein thrombosis of the lower extremities, one in every three patients will develop chronic DVT and post-thrombotic sequelae within 2years; these sequelae are severe in approximately $20 \%$ of cases and produce considerable socioeconomic consequences. In patients with associated post-phlebitic syndrome after specific therapy for DVT, patient should be advised to wear compression stocking which is the only proven therapy of benefit in this group of patients. The risk of post-phlebitic syndrome is not prevented by the long-term anticoagulant therapy. In patients with recurrent DVT, the presence of occult malignancy and myeloproliferative disorder has to be excluded.

\section{Conclusion}

Chronic recurrent DVT of the lower extremities is a significant socioeconomic problem, since it incurs a cost of at least \$200million annually to the United States health care, in addition to causing detriment upon patient quality of life and disability. So far, testing for inherited hypercoagulable defects has been found to have little value with respect to prophylactic strategies even though inherited hypercoagulable state is a major risk factor for recurrence. As there are no randomized controlled trials, research is needed with interventions such as thrombolysis, endovascular surgery, addition of aspirin or extended or intensive courses of anticoagulation, to practice evidence based medicine. In conclusion, treatment based on evidence based guideline is very sparse in management of recurrent DVT and further work is needed in this regard.

\section{Acknowledgements}

None.

\section{Conflict of interest}

The author declares no conflict of interest.

\section{References}

1. White RH. The epidemiology of venous thromboembolism. Circulation. 2003;107(23 Suppl 1):I4-18

2. Poulikidis KP, Gasparis AP, Labropoulos N. Prospective analysis of incidence, extent and chronicity of lower extremity venous thrombosis. Phlebology. 2014;29(1):37-42.

3. Dep A, Concannon E, Mc Hugh SM, et al. Paget-Schrotter syndrome and complications of management. BMJ Case Rep. 2013;2013.

4. Sajid MS, Ahmed N, Desai M, et al. Upper limb deep vein thrombosis: a literature review to streamline the protocol for management. Acta Haematol. 2007;118(1):10-18.

5. Rose SS, Ali Y, Kumar A, et al. Deep venous thrombosis caused by inferior vena cava atresia and hereditary thrombophilia. Am J Med Sci. 2009;337(1):67-70.

6. Heit JA, Mohr DN, Silverstein MD, et al. Predictors of recurrence after deep vein thrombosis and pulmonary embolism: a population-based cohort study. Arch Intern Med. 2000;160(6):761-768.

7. Bauer KA. The thrombophilias: well-defined risk factors with uncertain therapeutic implications. Ann Intern Med. 2001;135(5):367-373.

8. Wiszniewski A, Bykowska K, Bilski R, et al. Prevalence rate for inherited thrombophilia in patients with chronic and recurrent venous leg ulceration. Wound Repair Regen. 2011;19(5):552-558. 
9. Hansson PO, Sörbo J, Eriksson H. Recurrent venous thromboembolism after deep vein thrombosis: incidence and risk factors. Arch Intern Med. 2000;160(6):769-774.

10. Simioni P, Prandoni P, Lensing AW, et al. The risk of recurrent venous thromboembolism in patients with an Arg506--> Gln mutation in the gene for factor V (factor V Leiden). N Engl J Med. 1997;336(6):399-403.

11. Ridker PM, Miletich JP, Stampfer MJ, et al. Factor V Leiden and risks of recurrent idiopathic venous thromboembolism. Circulation. 1995;92(10):2800-2802.

12. Kyrle PA, Minar E, Hirschl M, et al. High plasma levels of factor VIII and the risk of recurrent venous thromboembolism. $N$ Engl J Med. 2000;343(7):457-462.

13. Dentali F, Ageno W, Bozzato S, et al. Role of factor V Leiden or G20210A prothrombin mutation in patients with symptomatic pulmonary embolism and deep vein thrombosis: a meta-analysis of the literature. $J$ Thromb Haemost. 2012;10(4):732-737.

14. Eichinger S, Stümpflen A, Hirschl M, et al. Hyperhomocysteinemia is a risk factor of recurrent venous thromboembolism. Thromb Haemost. 1998;80(4):566-569.

15. De Stefano V, Martinelli I, Mannucci PM, et al. The risk of recurrent venous thromboembolism among heterozygous carriers of the G20210A prothrombin gene mutation. Br J Haematol. 2001;113(3):630-635.

16. Eichinger S, Weltermann A, Mannhalter C, et al. The risk of recurrent venous thromboembolism in heterozygous carriers of factor $\mathrm{V}$ Leiden and a first spontaneous venous thromboembolism. Arch Intern Med. 2002;162(20):2357-2360.

17. Lindmarker P, Schulman S, Sten-Linder M, et al. The risk of recurrent venous thromboembolism in carriers and non-carriers of the G1691A allele in the coagulation factor $\mathrm{V}$ gene and the G20210A allele in the prothrombin gene. DURAC Trial Study Group. Duration of Anticoagulation. Thromb Haemost. 1999;81(5):684-689.

18. Christiansen SC, Cannegieter SC, Koster T, et al. Thrombophilia, clinical factors, and recurrent venous thrombotic events. JAMA. 2005;293(19):2352-2361.

19. Kyrle PA. How I treat recurrent deep-vein thrombosis. Blood. 2016;127(6):696-702.

20. Gaitini D. Current approaches and controversial issues in the diagnosis of deep vein thrombosis via duplex Doppler ultrasound. J Clin Ultrasound. 2006;34(6):289-297.

21. Andrews EJ, Fleischer AC. Sonography for deep venous thrombosis: current and future applications. Ultrasound Q. 2005;21(4):213-225.

22. Dermody M, Alessi-Chinetti J, Iafrati MD, et al. The utility of screening for deep venous thrombosis in asymptomatic, non-ambulatory neurosurgical patients. J Vasc Surg. 2011;53(5):1309-1315.

23. Pennell RC, Mantese VA, Westfall SG. Duplex scan for deep vein thrombosis-defining who needs an examination of the contralateral asymptomatic leg. J Vasc Surg. 2008;48(2):413-416.

24. Moed BR, Miller JR, Tabaie SA. Sequential duplex ultrasound screening for proximal deep venous thrombosis in asymptomatic patients with acetabular and pelvic fractures treated operatively. J Trauma Acute Care Surg. 2012;72(2):443-447.

25. O'Shaughnessy AM, Fitzgerald DE. Natural history of proximal deep vein thrombosis assessed by duplex ultrasound. Int Angiol. 1997;16(1):45-49.

26. Pomero F, Dentali F, Borretta V, et al. Accuracy of emergency physician-performed ultrasonography in the diagnosis of deep-vein thrombosis: a systematic review and meta-analysis. Thromb Haemost 2013;109(1):137-145.
27. Meissner MH. Duplex follow-up of patients with DVT: does it have clinical significance? Semin Vasc Surg. 2001;14(3):215-221.

28. Linkins LA, Stretton R, Probyn L, et al. Interobserver agreement on ultrasound measurements of residual vein diameter, thrombus echogenicity and Doppler venous flow in patients with previous venous thrombosis. Thromb Res. 2006;117(3):241-247.

29. Labropoulos N, Waggoner T, Sammis W, et al. The effect of venous thrombus location and extent on the development of post-thrombotic signs and symptoms. J Vasc Surg. 2008;48(2):407-412.

30. Labropoulos N, Gasparis AP, Tassiopoulos AK. Prospective evaluation of the clinical deterioration in post-thrombotic limbs. J Vasc Surg. 2009;50(4):826-830.

31. Turpie AG, Levine MN, Hirsh J, et al. Tissue plasminogen activator (rtPA) vs heparin in deep vein thrombosis. Results of a randomized trial. Chest. 1990;97(4 Suppl):172S-175S.

32. Watz R, Savidge GF. Rapid thrombolysis and preservation of valvular venous function in high deep vein thrombosis. A comparative study between streptokinase and heparin therapy. Acta Med Scand. 1979;205(4):293-298.

33. Gagne P, Khoury T, Zadeh BJ, et al. A Multicenter, Retrospective Study of the Effectiveness of the Trellis- 8 system in the treatment of proximal lower-extremity deep vein thrombosis. Ann Vasc Surg. 2015;29(8):16331641.

34. Tichelaar VY, Brodin EE, Vik A, et al. A retrospective comparison of ultrasound-assisted catheter-directed thrombolysis and catheter-directed thrombolysis alone for treatment of proximal deep vein thrombosis. Cardiovasc Intervent Radiol. 2016;39(8):1115-1121.

35. Watson L, Broderick C, Armon MP. Thrombolysis for acute deep vein thrombosis. Cochrane Database Syst Rev. 2014;(1):CD002783.

36. Prandoni P, Lensing AW, Cogo A, et al. The long-term clinical course of acute deep venous thrombosis. Ann Intern Med. 1996;125(1):1-7.

37. Marik PE, Cavallazzi R. Extended Anticoagulant and Aspirin Treatment for the Secondary Prevention of Thromboembolic Disease: A Systematic Review and Meta-Analysis. PLoS One. 2015;10(11):e0143252.

38. Patel NH, Stookey KR, Ketcham DB, et al. Endovascular management of acute extensive iliofemoral deep venous thrombosis caused by MayThurner syndrome. J Vasc Interv Radiol. 2000;11(10):1297-1302.

39. Jabri H, Mukherjee S, Sanghavi D, et al. Bilateral Upper Extremity DVT in a 43-Year-Old Man: Is It Thoracic Outlet Syndrome?! Case Rep Med. 2014;2014:758010.

40. Rosales A, Sandbaek G, Jørgensen JJ. Stenting for chronic post-thrombotic vena cava and iliofemoral venous occlusions: mid-term patency and clinical outcome. Eur J Vasc Endovasc Surg. 2010;40(2):234-240.

41. Gu YQ, Zhang J, Qi LX, et al. [Treatment of severe acute deep venous thrombosis in lower extremity]. Zhonghua Yi Xue Za Zhi. 2009;89(45):3186-3188.

42. Grommes J, Strijkers R, Greiner A, et al. Safety and feasibility of ultrasound-accelerated catheter-directed thrombolysis in deep vein thrombosis. Eur J Vasc Endovasc Surg. 2011;41(4):526-532.

43. Botella FG, Labios Gomez M, Portoles Reparaz O, et al. [New advances in the knowledge on post-thrombotic syndrome]. Anales de medicina interna. 2003;20(9):483-492.

44. Pesavento R, Bernardi E, Concolato A, et al. Post thrombotic syndrome. Semin Thromb Hemost. 2003;32(7):744-751. 